\title{
Clast collision frequency as an indicator of glacier sliding rate
}

\author{
Urs H. Fischer, " Garry K. C. Clarke \\ Department of Earth and Ocean Sciences, University of British Columbia, Vancouver, British Columbia V6 124, Canada
}

\begin{abstract}
The mechanical interaction between a glacier and subglacial sediment can be observed using an instrumented rod that we refer to as the UBC ploughmeter. For clast-rich sediments, the rate of collision between clasts and a rod dragged through these sediments should be related to the glacier sliding rate. By assuming that proglacial measurements of sediment granulometry represent the subglacial granulometry of Trapridge Glacier, Yukon Territory, Canada, we have used ploughmeter results to obtain an estimated sliding rate of $\sim 45 \mathrm{~mm} \mathrm{~d}^{1}$, in good agreement with known rates. In addition, for a subglacial material treated as a solid-liquid dispersion having a linear viscous rheology, the force of collision experienced by the rod should be proportional to the effective sediment viscosity. Our estimate of $\sim 2.0 \times 10^{10} \mathrm{~Pa}$ s agrees well with previously derived values.
\end{abstract}

\section{INTRODUCTION}

Glaciers and ice sheets on soft beds flow by some combination of ice creep, basal sliding and subglacial sediment deformation (Alley, 1989). It remains unclear what processes control the partitioning between sliding and sediment deformation, and without new instruments and extensive subglacial measurements it is doubtful that this question can be settled. To this end, we have developed an instrument, the UBC ploughmeter, to sense the mechanical interaction between a glacier and its bed (Fischer and Clarke, 1994). The essential idea is that a steel rod, with its tip protruding into subglacial sediment, will bend elastically as the tip is dragged through the sediment by movement of the overlying ice (Fig. la), much as a stick bends if it is used to inscribe lines on a sandy beach. The main body of the rod is embedded in ice, while the tip is instrumented with strain gauges that respond to bending in two mutually orthogonal directions (Fig. 1b). Detailed information on the construction, calibration, installation and theory of this device is given in Fischer and Clarke (1994). For the purpose of the present paper it is only necessary to understand that the ploughmeter measures the bending moment acting near the rod tip and that this moment is influenced by the depth of penetration, the sliding rate and bed properties.

For a homogeneous, fine-grained substrate, modelled as a linear viscous fluid having viscosity $\mu$, the force per unit length $F$ acting near the tip is approximated by

$$
F=\mu U L
$$

where $U$ is the relative velocity between rod and sediment (here assumed to equal the basal sliding rate) and $L$ is a characteristic length scale (Fischer and Clarke, 1994). For a

" Present address: Versuchsanstalt für Wasserbau, Hydrologie und Glaziologie, Eidgenössische Technische Hochschule, CH-8092 Zürich, Switzerland.

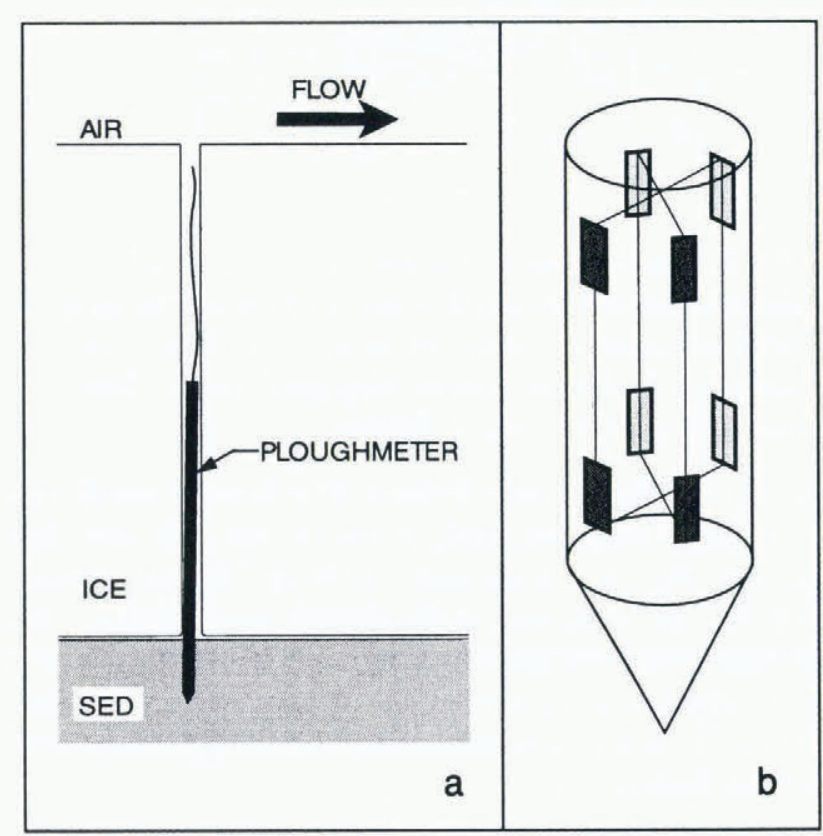

Fig. 1. (a) Schematic diagram of ploughmeter operation. (b) Arrangement of strain gauges near the tip of the steel rod.

heterogeneous or clast-rich sediment, a purely linear viscous assumption is inappropriate and the concepts of mean free path and clast-collision frequency suggest themselves. The mean free path is the typical travel distance between clast collisions; collision frequency is the inverse of the typical time interval between collisions. Potential complicating factors are temporal variations in sliding rate and spatial variations in bed granulometry.

\section{OBSERVATIONS}

During the 1991 summer field season, two ploughmeters were installed at the bed of Trapridge Glacier, Yukon Terri- 
tory, Canada. The location and a detailed description of the Trapridge Glacier study area are given in Clarke and Blake (1991). Figure 2 shows roughly $60 \mathrm{~d}$ of observation for ploughmeter 91PL02. During the course of these measurements, we collected data at 20 minute intervals. We estimate that the ploughmeter was inserted $\sim 0.15 \mathrm{~m}$ into the basal sediment. Figure $2 \mathrm{a}$ indicates the bending force applied to the tip of the ploughmeter, and Figure $2 b$ indicates the azimuth of the force with respect to the internal coordinates of the ploughmeter. During the entire observation period,

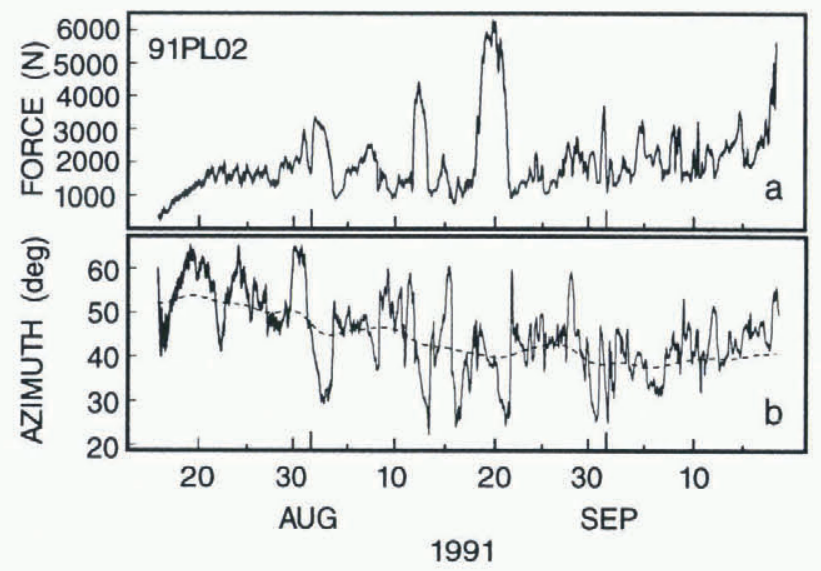

Fig. 2. Data from ploughmeter 91PL02. (a) Force record indicating force applied to the tip of the ploughmeter. (b) Azimuth of the force with respect to the internal coordinates of the ploughmeter. Dashed line represents the slowly varying azimuth component (see text for details).

both the force record and the azimuth record display a jagged appearance with rapid and large fluctuations. Variations in azimuth of the applied force could result from rotation of the ploughmeter about its long axis, from temporal variations in the direction of glacier flow, or from translational motion in a direction perpendicular to the glacier flow. Because we believe that ploughmeters are firmly gripped by glacier ice (Fischer and Clarke, 1994), it is unlikely that they will undergo large and rapid back-and-forth rotations within a borehole (Fig. 2 b). To further interpret the data, we postulate that azimuth variations due to changing glacier flow directions are generally long-term (cycles of $1 \mathrm{~d}$ or more) and that variations due to cross-flow motion are generally short-term (cycles of on the order of hours or less). In our analysis, we used a Gaussian low-pass filter (cut-off frequency $\approx 0.074 \mathrm{~d}^{-1}$ ) to extract the slowly varying component of the azimuth time series (dashed line in Fig. 2b) and attributed this slowly varying component to changes in the glacier flow direction. Any remaining high-frequency disturbance on the azimuth record indicates non-zero crossflow motion. By aligning the slowly varying azimuth component with the glacier flow direction, we can decompose the net force values (Fig. 2a) into down-flow and cross-flow components (Fig. 3). To effect this decomposition we assume that the principal direction of ploughmeter motion is downglacier.

\section{INTERPRETATION}

The jagged appearance of the force record (Fig. 2a) and the lack of an apparent correlation between the records of

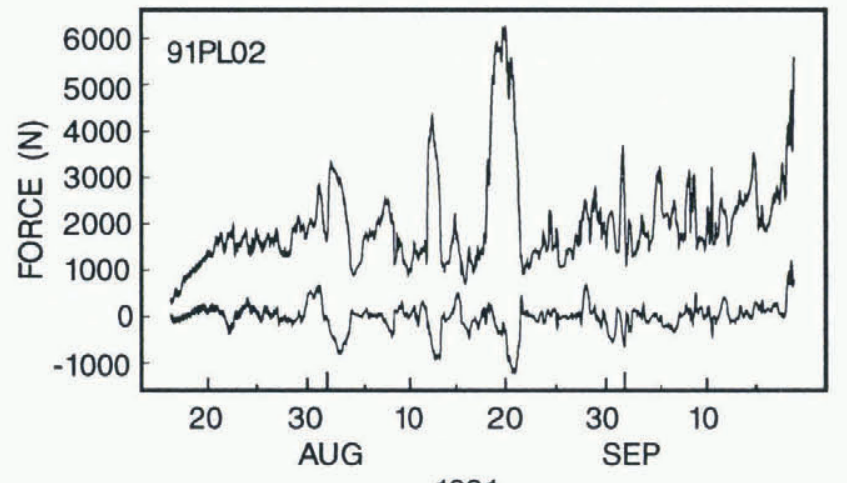

1991

Fig. 3. Decomposition into down-flow (upper trace) and cross-flow (lower trace) force components of the data from ploughmeter 91PLO2 shown in Figure 2.

down-flow and cross-flow force components (Fig. 3) suggest that ploughmeter 91PL02 is interacting with a clast-rich area of the glacier bed. The basis for this suggestion lies in the idea that the spikes in the data record are the result of the ploughmeter colliding with individual clasts as it is dragged through basal material. An alternative interpretation is that the spikes reflect interactions with groups or "bridges" of grains. During shearing of a granular material, grain alignment can become sufficiently coaxial that the resulting bridges support anomalously high shear and normal stresses (Hooke and Iverson, 1995). However, as these bridges fail by crushing of the particles, there is a tendency for the material to develop a self-similar size distribution in which particles of the same size are separated from each other (Sammis and others, 1987). In such a spatial arrangement of particles, intergranular stresses are more uniformly distributed and the tendency for mechanical heterogeneity is minimized (Iverson and others, 1995). Hence, because the particle-size distribution for Trapridge sediment was shown to approximate a fractal distribution (Fischer, 1995) we prefer the interpretation of a ploughmeter colliding with individual clasts.

To support our idea we simulated collisions between a ploughmeter and individual clasts as the ploughmeter moves through a heterogeneous subglacial sediment. We developed a simple numerical model to describe the response of a ploughmeter to such forcing by interaction with a synthetic till. For simplicity, we only considered nearest neighbour collisions. The model till was constructed by filling a volume with spheres of different sizes. The spheres represent clasts in a matrix of fine-grained solids and water-filled voids. We assumed a porosity of 0.3 and a clast-size distribution that is based on the granulometry of a basal till from Trapridge Glacier (Clarke, 1987). Only clasts of the seven largest size classes were included in the model, corresponding to spheres of diameters in the range 4-32 $\mathrm{mm}$ (Table 1). Grains of smaller size were assumed to form the matrix of fines. To achieve a random yet spatially fractal distribution of clasts within the till, the spheres were arranged inside the volume using a method similar to the way a dense concrete is created. As a first step, the largest spheres were randomly assembled. Subsequently, spheres of the next smaller size class were placed into the voids left between the larger ones. This second stage was then followed by adding yet smaller spheres which fill the gaps between the first and second sets of spheres. By recursively placing smaller spheres in between the larger spheres, this filling process was continued until the 
Table 1. Parameters for model till

\begin{tabular}{ccccc} 
Grain diameter Radius of sphere & $\begin{array}{c}\text { Number per } \\
\text { unit volume }\end{array}$ & Typical distance Volume fraction \\
$\varphi$ & $R$ & $N$ & $d$ & $c$ \\
& $\mathrm{~mm}$ & $\mathrm{~m}_{\mathrm{S}}^{-3}$ & $\mathrm{~mm}$ & \\
\hline-5.0 & 16.0 & 4279 & 61.59 & 0.073 \\
-4.5 & 11.31 & 7146 & 51.98 & 0.047 \\
-4.0 & 8.0 & 14326 & 41.17 & 0.035 \\
-3.5 & 5.66 & 34833 & 30.39 & 0.032 \\
3.0 & 4.0 & 86162 & 22.64 & 0.028 \\
-2.5 & 2.83 & 218488 & 16.77 & 0.025 \\
-2.0 & 2.0 & 538944 & 12.29 & 0.023 \\
\hline
\end{tabular}

smallest size class had been reached. The resulting cascades of smaller and smaller spheres filling the gaps between larger ones is similar to the packing of circles in the fractal Apollonian gasket (Fig. 4).

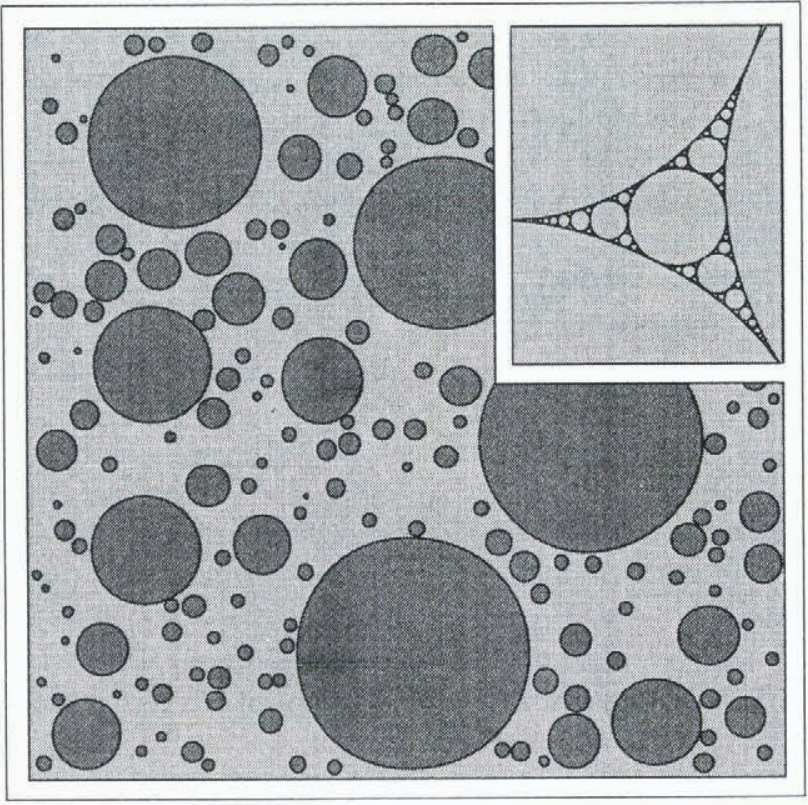

Fig. 4. Cross-sectional cut through the model till. The area shown is $0.1 \mathrm{~m}$ by $0.1 \mathrm{~m}$ in size. The light shaded regions represent the matrix of fine-grained solids and water-filled voids. Inset shows a mathematically perfect Apollonian gasket (adapted from Mandelbrot, 1983, p. 170).

The transverse motion of a vertical cylinder through the volume represents the ploughmeter being dragged through subglacial material (Fig. 5). The cylinder was taken to have a radius of $16 \mathrm{~mm}$ (Table 2) which is consistent with the crosssectional dimension of the ploughmeter (Fischer and Clarke, 1994). To account for the insertion depth of the ploughmeter into basal sediment, the height of the model till

Table 2. Parameters for "clast collision" model

\begin{tabular}{lccc}
\hline \multicolumn{1}{c}{ Parameter } & Symbol & Value & Unit \\
\hline Radius of cylinder & $R_{\mathrm{C}}$ & 16.0 & $\mathrm{~mm}$ \\
Height of till volume & $h_{\mathrm{T}}$ & 0.15 & $\mathrm{~m}$ \\
Porosity of till & $n$ & 0.3 & \\
& & & \\
\hline
\end{tabular}

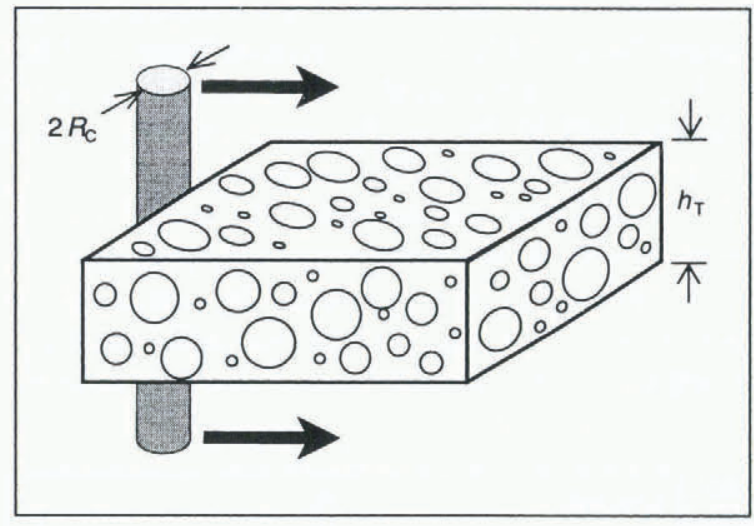

Fig. 5. Schematic diagram of "clast collision" model. The vertical cylinder represents the ploughmeter moving through subglacial sediment.

volume was assumed to be $0.15 \mathrm{~m}$ (Table 2). We view the spheres as being suspended in a viscous medium. Thus, we treat the synthetic till as a solid-liquid dispersion and calculate the forces on the cylinder as it moves through this dispersion. Two parts contribute to these forces. The drag on the cylinder is calculated using Equation (1). This force contribution has only a component in the down-flow direction and, assuming that the glacier slides at a steady rate, is constant with time. The calculation of the forces that arise from collisions with the spheres is based on Stokes' law

$$
F=6 \pi \mu R U
$$

which describes the total drag of a sphere of radius $R$ moving through a viscous fluid at velocity $U$ (Stokes, 1851). The idea is that after impacting, the cylinder deflects the spheres to the side, thereby pushing them through the viscous medium. Because impacts are likely to be oblique with respect to the direction of motion of the cylinder, these forces have down-flow and cross-flow components.

Instead of considering the motion of a cylinder through the fluid we change the frame of reference and analyze the uniform flow around the cylinder located at the origin (Fig. 6). Fluid flow is assumed to be directed along the $x$ axis. The spheres in the model till move at the fluid velocity and follow a trajectory that corresponds to a streamline. Collision between the cylinder and a given sphere occurs when the streamline is such that the sphere is not carried far enough around to clear the cylinder. If the velocity of the

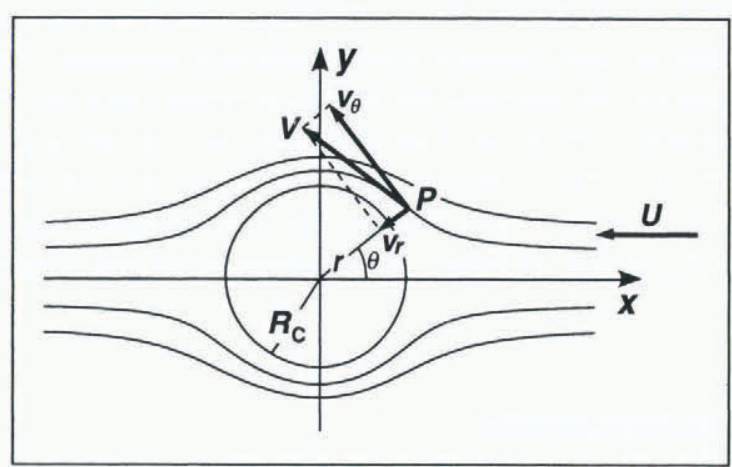

Fig. 6. Uniform fluid flow around a cylinder (adapted from Eskinazi, 1962, p. 288). 
fluid at infinity is $U$ then the velocity field at any point $P$ can be expressed as (e.g. Eskinazi, 1962, p. 288)

$$
\begin{aligned}
& v_{r}=U\left[\left(\frac{R_{\mathrm{C}}}{r}\right)^{2}-1\right] \cos \theta \\
& v_{\theta}=U\left[\left(\frac{R_{\mathrm{C}}}{r}\right)^{2}-1\right] \sin \theta
\end{aligned}
$$

where $R_{\mathrm{C}}$ is the radius of the cylinder, $r$ is the length of the position vector for point $P$, and $\theta$ denotes the angle between $r$ and the direction of fluid flow at infinity (Fig. 6). The total velocity in the flow field is $V^{2}=v_{r}^{2}+v_{\theta}^{2}$.

When the cylinder and the sphere are in contact, $r$ is replaced by the sum of the radii of the cylinder and the sphere. It is the radial velocity component $v_{r}$ that causes the sphere to be deflected from its original streamline. In order to calculate the force components in the down-flow and cross-flow directions we therefore have to decompose $v_{r}$ into its $x$ and $y$ components (Fig. 7),

$$
\begin{aligned}
& v_{x}=v_{r} \cos \theta \\
& v_{y}=v_{r} \sin \theta .
\end{aligned}
$$

Hence, substituting Equation (3a) into Equation (4) yields

$$
\begin{aligned}
& v_{x}=U\left[\left(\frac{R_{\mathrm{C}}}{R_{\mathrm{C}}+R_{\mathrm{S}}}\right)^{2}-1\right] \cos ^{2} \theta \\
& v_{y}=U\left[\left(\frac{R_{\mathrm{C}}}{R_{\mathrm{C}}+R_{\mathrm{S}}}\right)^{2}-1\right] \sin \theta \cos \theta
\end{aligned}
$$

where $R_{\mathrm{S}}$ denotes the radius of the sphere. With reference to Figure 7 we recognize that $\cos \theta=x /\left(R_{\mathrm{C}}+R_{\mathrm{S}}\right)$ and $\sin \theta=y /\left(R_{\mathrm{C}}+R_{\mathrm{S}}\right)$ and thus obtain two equations that describe the motion of the sphere around the cylinder along the $x$ and $y$ directions, respectively,

$$
\begin{aligned}
& v_{x}=\frac{\mathrm{d} x}{\mathrm{~d} t}=\frac{U x^{2}(t)}{\left(R_{\mathrm{C}}+R_{\mathrm{S}}\right)^{2}}\left[\left(\frac{R_{\mathrm{C}}}{R_{\mathrm{C}}+R_{\mathrm{S}}}\right)^{2}-1\right] \\
& v_{y}=\frac{\mathrm{d} y}{\mathrm{~d} t}=\frac{U x(t) y(t)}{\left(R_{\mathrm{C}}+R_{\mathrm{S}}\right)^{2}}\left[\left(\frac{R_{\mathrm{C}}}{R_{\mathrm{C}}+R_{\mathrm{S}}}\right)^{2}-1\right] .
\end{aligned}
$$

The temporal evolution of the force components in the down-flow and cross-flow directions that arise as the sphere is being deflected from its original streamline can now be easily calculated by substituting Equations (6) into Equation (2). However, these force calculations only ac-

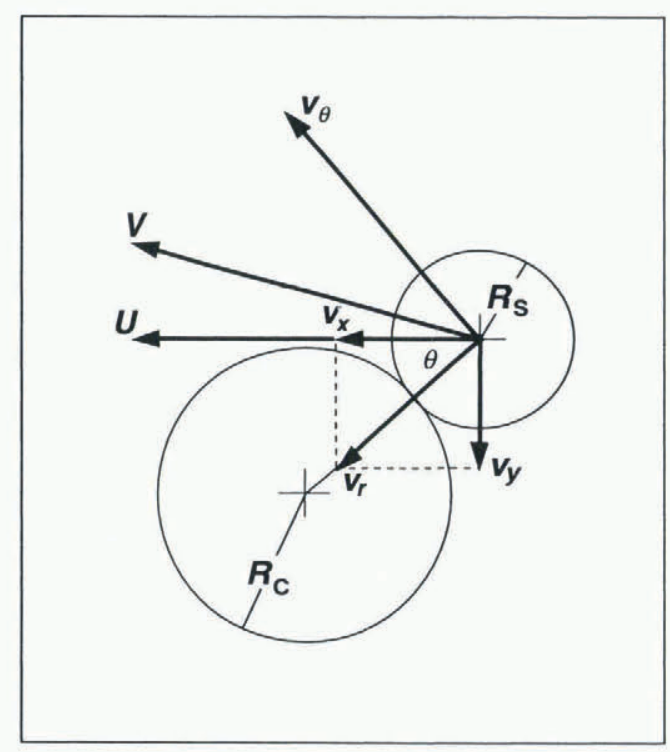

Fig. 7. Velocity components on a sphere colliding with a cylinder. count for interactions that take place while the cylinder and the sphere are in contact, and neglect any forces that are exchanged hydrodynamically before the collision has actually occurred. This "pre-collision" hydrodynamic interaction was simulated by ramping up the force components proportionally to the distance by which the streamline is deflected from horizontal (Fig. 6).

In an attempt to describe the viscous behaviour of the synthetic till, it is useful to treat the solid-liquid dispersion as an effective medium to which an effective viscosity can be ascribed. If a small number of particles is added to a liquid, its apparent viscosity increases as described by the relation (Einstein, 1906, 1911)

$$
\mu=\mu_{0}\left(1+\frac{5}{2} c\right)
$$

where $c$ is the volume fraction of the added particles $(c \ll 1), \mu$ is the apparent viscosity of the dispersion, and $\mu_{0}$ that of the suspending medium. Following the principles of effective medium theory (Van de Ven, 1989, p. 539), we computed an effective viscosity for the model till by successive substitution. Algorithmically, this amounts to the following procedure. We start with the matrix of fine-grained solids and water-filled voids and treat this initial dispersion as a homogeneous material to which we ascribe a background viscosity $\mu_{\mathrm{B}}$. Subsequently, we add the spheres of the smallest size class to this material and calculate the increase in apparent viscosity according to Equation (7). We then regard the resulting "effective" dispersion with this new effective viscosity as the homogeneous material for the succeeding substitution step. This process continues until the spheres of the largest size class have been added to the dispersion. The associated final effective viscosity is what we might refer to as bulk viscosity of the synthetic till and is analogous to the apparent viscosity of subglacial sediments inferred from in situ or laboratory measurements (Humphrey and others, 1993; Fischer and Clarke, 1994; Porter and others, 1997). However, when analyzing the drag on a sphere of a given size class we substitute the viscosity that was calculated for this size class into Equation (2). The reason for not substituting the bulk viscosity is that the sphere moves in a "cage" formed by neighbouring spheres of the next larger size class. Because the typical distance of these neighbouring spheres is 3-4 times larger than the size of the sphere under consideration (Table 1), its motion is only affected by spheres of the same and smaller size classes. The foregoing thinking represents a standard application of effective medium theory (Van de Ven, 1989, p. 539) and is not original to this work.

As a first simulation (Fig. 8), we substituted a background viscosity $\mu_{\mathrm{B}}=1.0 \times 10^{10} \mathrm{~Pa}$ s and a fluid velocity $U=0.04 \mathrm{~m} \mathrm{~d}^{-1}$ into our model equations. This background viscosity corresponds to a bulk viscosity of the synthetic till $\mu=2.06 \times 10^{10} \mathrm{Pas}$ which approximates values inferred for Trapridge sediment (Fischer and Clarke, 1994). In our analysis, the fluid velocity is equivalent to the translational velocity of the cylinder $\left(U=U_{\mathrm{C}}\right)$ and represents the differential velocity between ploughmeter and sediment which can be seen as a proxy for the glacier sliding rate. A value of $0.04 \mathrm{~m} \mathrm{~d}^{-1}$ is a typical basal sliding velocity measured at Trapridge Glacier (Blake and others, 1994).

Comparison of Figures 3 and 8 shows that the records generated using our model display many of the features seen in the real data. However, we note the lack of "broad" spikes 


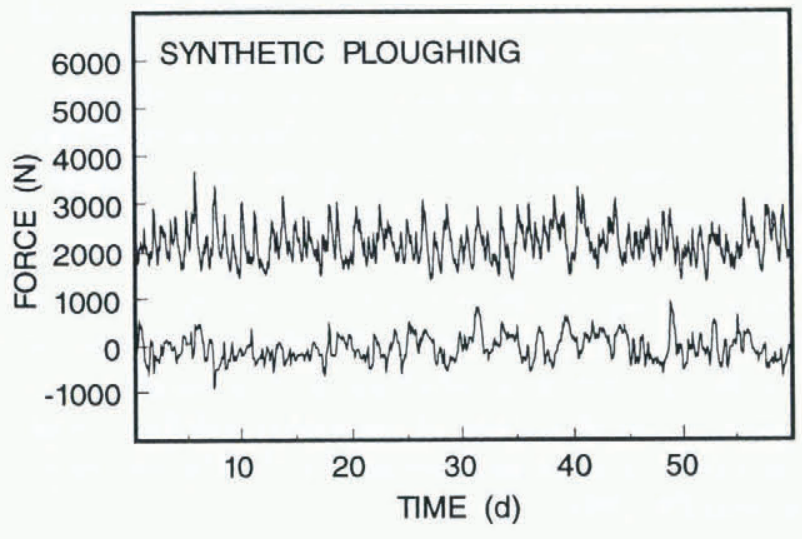

Fig. 8. Synthetically generated records of down-flow (upper trace) and cross-flow (lower trace) force components. Note the similarity to Figure 3.

in the synthetically generated records of down-flow and cross-flow force components (Fig. 8). The absence of broad spikes could reflect the fact that clasts larger than $32 \mathrm{~mm}$ in diameter were not included in our model till (Table 1). Even though the largest clasts in a basal till sample from Trapridge Glacier (Clarke, 1987) were of this size, evidence from the ablation till in the forefield of the glacier indicates that the basal sediment layer contains clasts as large as cobbles and boulders. The upper limit of clast diameter of $32 \mathrm{~mm}$, as determined by sedimentological size analysis (Clarke, 1987), is likely to be artificial and results from a bias towards smaller clasts during the sampling procedure. The sampling involved filling a $0.008 \mathrm{~m}^{3}$ pail with "typical till", excluding material too large to shovel (Clarke, 1987). Upon closer inspection of Figure 3, we see that collisions with larger clasts seem to have occurred mainly during the interval 30 August-22 September, and that broad spikes are mostly absent during the remaining time period. For this reason we base the analyis in the following section mainly on the second half of this force record.

\section{ESTIMATION OF BASAL SLIDING RATE AND SEDIMENT VISCOSITY}

In the above section we have shown that the jagged appearance of the force and azimuth record of ploughmeter 91PL02 (Fig. 2) can be interpreted as the ploughmeter interacting with a clast-rich sediment bed whereby the spikes in the data record indicate collisions with clasts. If we assume that the bed granulometry is spatially homogeneous and that temporal variations in sliding rate are negligible, the velocity with which the ploughmeter moves through the sediment is proportional to the rate of collision with clasts. In addition, assuming a linear viscous rheology for the subglacial sediment layer, a change in till viscosity and ploughmeter velocity results in a proportional change in the amplitude of the force records (Equations (1) and (2)). Therefore, by comparing the frequencies and the total power contained in the measured and synthetically generated force records (Figs 3 and 8), we are able to estimate the glacier sliding rate and an effective sediment viscosity.

Suppose that ploughmeter-sediment interactions yield a response $r(t)$. The frequency content of this response can be represented by the power spectrum $S(f)$ given by

$$
S(f)=\left\langle R(f) R(f)^{*}\right\rangle
$$

where $R(f)$ is the Fourier transform of $r(t)$ and angular brackets denote time averaging. If $r(t)$ is sampled at $N$ points over a time period $T$, the total power of this time series is given by

$$
\left\langle[r(t)]^{2}\right\rangle=\frac{1}{T} \int_{0}^{T}|r(t)|^{2} \mathrm{~d} t \approx \frac{1}{T} \sum_{i=0}^{N-1}\left|r_{i}\right|^{2} .
$$

A reasonable starting-point is to generate synthetic records of the force components in the down-flow and cross-flow directions for some plausible values of till viscosity $\mu^{\mathrm{s}}$ and glacier sliding rate $U^{\mathrm{s}}$ (e.g. Fig. 8). We denote these simulated times series by $F_{x}^{\mathrm{s}}(t)$ and $F_{y}^{\mathrm{s}}(t)$. We can now relate these simulated time series to those that were obtained from our field measurements (Fig. 3). Presumably the values of till viscosity and sliding rate are different from those used in the simulation, hence

$$
\begin{aligned}
& F_{x}^{\mathrm{m}}(t)=\frac{\mu^{\mathrm{m}} U^{\mathrm{m}}}{\mu^{\mathrm{s}} U^{\mathrm{s}}} F_{x}^{\mathrm{s}}\left(U^{\mathrm{s}} t / U^{\mathrm{m}}\right) \\
& F_{y}^{\mathrm{m}}(t)=\frac{\mu^{\mathrm{m}} U^{\mathrm{m}}}{\mu^{\mathrm{s}} U^{\mathrm{s}}} F_{y}^{\mathrm{s}}\left(U^{\mathrm{s}} t / U^{\mathrm{m}}\right)
\end{aligned}
$$

where $\mu^{\mathrm{m}}$ and $U^{\mathrm{m}}$ are the values that we seek to determine. Note that a different sliding rate also causes the time axis to be rescaled because of a change in the rate of collision. Introducing the dimensionless ratios $\alpha=U^{\mathrm{m}} / U^{\mathrm{s}}$ and $\beta=\mu^{\mathrm{m}} / \mu^{\mathrm{s}}$ gives

$$
\begin{aligned}
& F_{x}^{\mathrm{m}}(t)=\alpha \beta F_{x}^{\mathrm{s}}(t / \alpha) \\
& F_{y}^{\mathrm{m}}(t)=\alpha \beta F_{y}^{\mathrm{s}}(t / \alpha) .
\end{aligned}
$$

Consequently, the power spectra of $F_{x}^{\mathrm{m}}(t)$ and $F_{y}^{\mathrm{m}}(t)$ are respectively related to those for the simulated time series $F_{x}^{\mathrm{s}}(t)$ and $F_{y}^{\mathrm{s}}(t)$ by

$$
\begin{aligned}
& S_{x}^{\mathrm{m}}(f)=\alpha^{4} \beta^{2} S_{x}^{\mathrm{s}}(\alpha f) \\
& S_{y}^{\mathrm{m}}(f)=\alpha^{4} \beta^{2} S_{y}^{\mathrm{s}}(\alpha f) .
\end{aligned}
$$

Here, we have made use of the Fourier transform similarity and addition theorems (e.g. Bracewell, 1986, p. 122). Further, the power in the time series $F_{x}^{\mathrm{m}}(t)$ and $F_{y}^{\mathrm{m}}(t)$ can be related to the power in the simulated time series as follows:

$$
\begin{aligned}
& \left\langle\left[F_{x}^{\mathrm{m}}(t)\right]^{2}\right\rangle=\alpha^{2} \beta^{2}\left\langle\left[F_{x}^{\mathrm{s}}(t / \alpha)\right]^{2}\right\rangle=\alpha^{2} \beta^{2}\left\langle\left[F_{x}^{\mathrm{s}}(t)\right]^{2}\right\rangle \\
& \left\langle\left[F_{y}^{\mathrm{m}}(t)\right]^{2}\right\rangle=\alpha^{2} \beta^{2}\left\langle\left[F_{y}^{\mathrm{s}}(t / \alpha)\right]^{2}\right\rangle=\alpha^{2} \beta^{2}\left\langle\left[F_{y}^{\mathrm{s}}(t)\right]^{2}\right\rangle .
\end{aligned}
$$

Note that the power is independent of the sampling interval (see Equation (9)). The implication of Equation (13) is that a simple multiplicative factor $\alpha^{2} \beta^{2}$ can be used to adjust the power levels of the simulated time series $F_{x}^{\mathrm{s}}(t)$ and $F_{y}^{\mathrm{s}}(t)$ to match the power levels of the observed time series $F_{x}^{\mathrm{m}}(t)$ and $F_{y}^{\mathrm{m}}(t)$. Combined, Equations (12) and (13) suggest a procedure for determining $\alpha$ and $\beta$ which leads to an evaluation of both till viscosity and sliding rate.

For both the data of ploughmeter 91PL02 (Fig. 3) and the synthetically generated data (Fig. 8), the records of the crossflow force component were analyzed by taking the Fourier transform (Press and others, 1992, p. 490), and the power spectral densities were estimated using the periodogram method (Press and others, 1992, p. 531). If plotted on $\log -\log$ scales, we see that both spectral energy functions, $S_{y}^{\mathrm{m}}(f)$ for the 91PL01 data (Fig. 9) and $S_{y}^{\mathrm{s}}(f)$ for the synthetic data (Fig. 10), show good power-law dependence on frequency over large parts of the spectra. Thus, the power spectra are of the form

$$
S(f) \sim f^{-b},
$$

which suggests that the rate of change of power is indepen- 


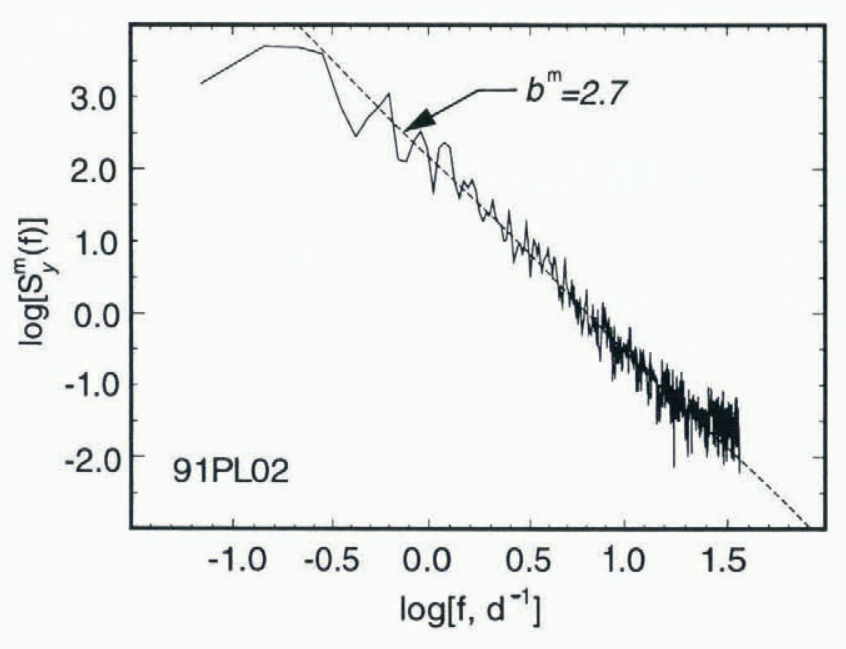

Fig. 9. Power spectral density function for the record of the cross-flow force component shown in Figure 3. The dashed line represents Equation (14) with $b^{\mathrm{m}}=2.7$.

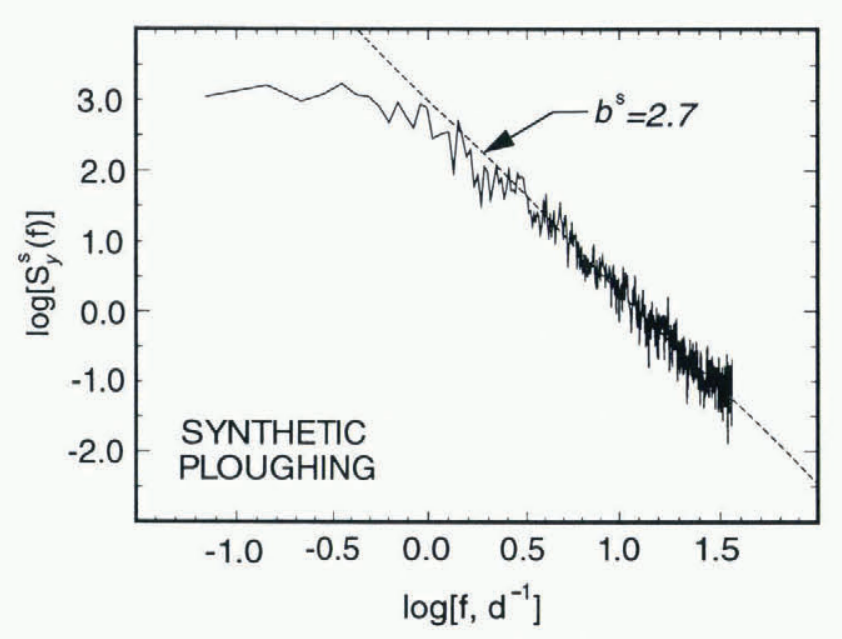

Fig. 10. Power spectral density function for the record of the cross-flow force component shown in Figure 8. The dashed line represents Equation (14) with $b^{\mathrm{s}}=2.7$.

dent of scale. Considering that the ploughmeter interacted with a sediment having a self-similar particle-size distribution (Fischer, 1995), we are not surprised to find that the frequency -amplitude statistics of the force records are fractal. The least-squares $\log -\log$ slopes from Equation (14) on the quasi-linear sections of the spectra give $b=2.7$ for both cases. Because of this power-law scaling behaviour, we can represent the two power spectra $S_{y}^{\mathrm{m}}(f)$ and $S_{y}^{\mathrm{s}}(f)$ in $\log -\log$ space as two straight lines and, after defining $\gamma=\alpha \beta$, rewrite Equation (12) as

$$
a^{\mathrm{m}}+b^{\mathrm{m}} \log f=\log \alpha^{2}+\log \gamma^{2}+a^{\mathrm{s}}+b^{\mathrm{s}} \log f
$$

where $b^{\mathrm{m}}$ and $b^{\mathrm{s}}$ are the slopes and $a^{\mathrm{m}}$ and $a^{\mathrm{s}}$ the intercepts of the power spectra for the measured and synthetic data series, respectively. The product $\gamma=\alpha \beta$ can be evaluated by comparing the power levels of $F_{x}^{\mathrm{m}}(t)$ with $F_{x}^{\mathrm{s}}(t)$ (Equation (13)). Now, Equation (15) can be solved to yield a value for $\alpha$. This operation corresponds to shifting one of the power spectra with respect to the other, and we use this approach to determine the till viscosity and sliding rate that will achieve optimal overlap of the two power spectra.

Table 3 summarizes the results of our simulations. Calculations were performed with a variety of different input
Table 3. Input and output of "clast collision" model

\begin{tabular}{|c|c|c|c|c|c|c|}
\hline \multicolumn{2}{|c|}{ Input } & \multicolumn{5}{|c|}{ Output } \\
\hline $\begin{array}{l}\text { Velocity of } \\
\text { cylinder }\end{array}$ & $\begin{array}{c}\text { Background } \\
\text { viscosity }\end{array}$ & & & & $\begin{array}{l}\text { Sliding } \\
\text { rate }\end{array}$ & $\begin{array}{c}\text { Till } \\
\text { viscosity }\end{array}$ \\
\hline$U_{\mathrm{C}}=U^{\mathrm{s}}$ & $\mu_{\mathrm{B}}$ & $\gamma^{2}$ & $\alpha^{2}$ & $\beta^{2}$ & $U^{\mathrm{m}}$ & $\mu^{\mathrm{mI}}$ \\
\hline $\mathrm{md}^{1}$ & $\times 10^{10} \mathrm{Pas}$ & & & & $\mathrm{md}^{-1}$ & $\times 10^{10} \mathrm{Pas}$ \\
\hline 0.02 & 1.0 & 4.471 & 2.264 & 0.045 & 0.0453 & 1.922 \\
\hline 0.04 & 1.0 & 1.122 & 1.072 & 0.989 & 0.0428 & 2.034 \\
\hline 0.06 & 1.0 & 0.487 & 0.769 & 0.907 & 0.0462 & 1.867 \\
\hline 0.08 & 1.0 & 0.274 & 0.577 & 0.907 & 0.0462 & 1.866 \\
\hline 0.04 & 2.0 & 0.281 & 1.014 & 0.522 & 0.0406 & 2.149 \\
\hline 0.02 & 2.0 & 1.118 & 2.269 & 0.466 & 0.0454 & 1.918 \\
\hline 0.02 & 5.0 & 0.179 & 2.132 & 0.198 & 0.0426 & 2.041 \\
\hline 0.04 & 0.5 & 4.488 & 1.132 & 1.871 & 0.0453 & 1.925 \\
\hline
\end{tabular}

values of the background viscosity and the velocity of the cylinder to demonstrate the robustness of the model. The velocity $U_{\mathrm{C}}$ was assumed to take on values of 20 to $80 \mathrm{~mm} \mathrm{~d}^{-1}$, the latter of which corresponds to the surface velocity of Trapridge Glacier. Viscosity values were taken to span an order of magnitude and were in the range $\mu_{\mathrm{B}}=0.5-5.0 \times 10^{10} \mathrm{~Pa}$ s. In all cases, we obtained consistent results for the predicted glacier sliding rate and till viscosity. The glacier sliding velocity of $\sim 45 \mathrm{~mm} \mathrm{~d}^{-1}$ is in excellent agreement with direct measurements of sliding at the base of Trapridge Glacier using a "drag spool" (Blake and others, 1994). Similarly, the value of the sediment viscosity of $\sim 2.0 \times 10^{10} \mathrm{Pas}$ agrees well with estimates that had previously been obtained for Trapridge sediment from a theoretical analysis of the forces on a ploughmeter (Fischer and Clarke, 1994).

\section{CONGLUDING DISGUSSION}

In this paper, we have demonstrated that if the bed granulometry is known, the collision frequency and collision force as indicated by a ploughmeter can be used to obtain estimates of the basal sliding velocity and effective sediment viscosity. Our analysis assumed temporal variations in sliding rate to be negligible, a linear viscous rheology of the basal material and a spatially uniform granulometry. In the case of Trapridge Glacier we were able to confirm our velocity estimate by comparing it with direct measurements. Support for our viscosity estimate is given by previously derived values for Trapridge sediment.

In our analysis we have assumed that the differential velocity between ploughmeter and sediment is equivalent to the basal sliding rate of the glacier. Because glacier sliding is commonly defined as the motion between the base of the ice and the top of the bed, we have essentially neglected the contribution of the basal motion that arises from bed deformation. Therefore, the question of how a deforming sediment layer affects the ploughmeter record has to be addressed. If we assume that part of the basal velocity is due to bed deformation, sediments near the ice-bed interface have a velocity component in the down-flow direction. Thus, the differential velocity between ploughmeter and surrounding sediment is generally less compared to the case where the ploughmeter is moving through a non-deforming material. Accordingly, for this case, where the ploughmeter 
is moving through an already shearing material, both the frequency of collision and the force of collision are reduced. We outline the implications for our model by comparing the mode of motion in which $100 \%$ of the basal velocity is due to glacier sliding to that in which the basal velocity is partitioned between 50\% glacier sliding (Blake and others, 1994) and $50 \%$ bed deformation in a $0.15 \mathrm{~m}$ thick deformable layer with a shear strain rate that is constant with depth. To obtain similar frequencies and power levels in the force records generated with the two modes of basal motion, the basal velocity of the $50 \%$ glacier-sliding $/ 50 \%$ bed-deformation mode has to be larger by approximately one-quarter than that of the $100 \%$ glacier-sliding mode. However, because half of this increased basal velocity is due to bed deformation, the sliding velocity is effectively reduced by about one-third. As a result, by neglecting sediment deformation in our analysis we are likely to overestimate the basal sliding rate.

We compared our velocity estimate with direct measurements of sliding using a drag spool. This device is suspended within the borehole close to the base of the glacier, and continuously measures the length of string paid out to an anchor in the bed (Blake and others, 1994). Thus, we have defined "sliding" in an operational manner, as the motion between the spool and the anchor. We recognize that this definition is not equivalent to that of "true" basal sliding and that measurements taken with a drag spool will place only an upper limit on glacier sliding, because the anchor is placed within deformable sediment; any deformation of the sediment lying between the anchor and the ice-sediment contact will introduce additional string extension. With an anchor insertion depth of $\sim 0.18 \mathrm{~m}$, a typical thickness of the deformable layer underneath Trapridge Glacier of $\sim 0.5 \mathrm{~m}$ (Blake and Clarke, 1989) and the assumption that the sediment velocity varies linearly with depth, about $5-10 \%$ of the measured displacement is due to sediment deformation (Fischer, 1995). Hence, both methods of estimating the glacier-sliding rate yield values that tend to be somewhat on the high side.

Complications to our analysis arise if the bed granulometry is unknown and the clast-collision frequency is observed to vary with time. In this case, two contrasting interpretations which can be regarded as end-member cases present themselves: (1) the sliding rate is constant and bed granulometry spatially inhomogeneous; (2) the bed granulometry is spatially homogeneous and the sliding rate varies temporally. In the latter case, sliding rate is proportional to the clast-collision frequency. Suppose that sliding initially occurs at some constant rate $U$. If at some subsequent time the sliding velocity changed to some new but constant value $U^{\prime}=\alpha U$, the time axis of the ploughmeter response would become rescaled as $t^{\prime}=t / \alpha$ (see Equations (10) and (11)). As a result, the new response has a power spectrum $\alpha^{2} S(\alpha f)$ (Equation (12)). Thus, the relative change in sliding rate $\alpha=U^{\prime} / U$ produces a proportional rescaling of the frequency axis. It may therefore prove possible to use plough- meters to observe seasonal variations in the basal sliding rate.

\section{ACKNOWLEDGEMENTS}

We dedicate this paper to our friend and colleague B.S. Waddington who was killed in an avalanche on Mt. Cerberus, 17 May 1996. This study was supported by grants from the Natural Sciences and Engineering Research Council of Canada and the University of British Columbia. We thank Parks Canada and the Government of the Yukon Territory for permission to conduct research in Kluane National Park. B. S. Waddington, E.W. Blake, D. B. Stone and T. Murray provided advice and assistance during our field program. Thanks are also due to R. LeB. Hooke, N. R. Iverson and N. F. Humphrey who read an earlier version of this manuscript and made some valuable comments.

\section{REFERENCES}

Alley, R. B. 1989. Water-pressure coupling of sliding and bed deformation: II. Velocity depth profiles. f. Glaciol., 35(119), 119-129.

Blake, E.W. and G. K. C. Clarke. 1989. In situ strain measurements beneath a surge-type glacier. [Abstract.] EOS, 70 43), 1084.

Blake, E.W., U. H. Fischer and G. K. C. Clarke. 1994. Direct measurement of sliding at the glacier bed. F. Glaciol., 40 (136), 595-599.

Bracewell, R. N. 1986. The Fourier transform and its applications. Second revised edition. New York, McGraw-Hill Book Co.

Clarke, G. K. C. 1987. Subglacial till: a physical framework for its properties and processes. J. Geophys. Res., 92 (B9), $9023-9036$.

Clarke, G. K. C. and E. W. Blake. 1991. Geometric and thermal evolution of a surge-type glacier in its quiescent state: Trapridge Glacier, Yukon Territory, Canada, 1969 89. J. Glaciol., 37 (125), 158-169.

Einstein, A. 1906. Eine neue Bestimmung der Moleküldimensionen. Ann. Phys., 19 (2), 289-306.

Einstein, A. 1911. Berichtigung zu meiner Arbeit: "Eine neue Bestimmung der Moleküldimensionen". Ann. Phys., 34 (3), $591-592$.

Eskinazi, S. 1962. Principles of fluid mechanics. Boston, MA, Allyn and Bacon, Inc.

Fischer, U.H. 1995. Mechanical conditions beneath a surge-type glacier. (Ph.D. thesis, University of British Columbia.

Fischer, U. H. and G. K. C. Clarke. 1994. Ploughing of subglacial sediment. 7. Glaciol., 40 (134), 97-106.

Hooke, R. LeB. and N. R. Iverson. 1995. Grain-size distribution in deforming subglacial tills: role of grain fracture. Geology, 23(1), 57-60.

Humphrey, N., B. Kamb, M. Fahnestock and H. Engelhardt. 1993. Characteristics of the bed of the lower Columbia Glacier, Alaska. J. Geophys. Res., 98 (B1), 837-846.

Iverson, N. R., T. S. Hooyer and R. LeB. Hooke. 1996. A laboratory study of sediment deformation: stress heterogeneity and grain-size evolution. Ann. Glaciol, 22, 167-175.

Mandelbrot, B. B. 1983. The fractal geometry of nature. Second edition. New York, W. H. Freeman and Co.

Porter, P. R., T. Murray and J. A. Dowdeswell. 1997. Sediment deformation and basal dynamics beneath a glacier surge front: Bakaninbreen, Svalbard. Ann. Glaciol., 24, $21-26$.

Press, W. H., S. A. Teukolsky, W.T. Vetterling and B. P. Flannery. 1992. Numerical recipes in FORTRA.N: the art of scientific computing. Second edition. Cambridge, Cambridge University Press.

Sammis, C., G. King and R. Biegel. 1987. The kinematics of gouge deformation. Pure Appl. Geophys., 125 (5), 777-812.

Stokes, G. G. 1851. On the effect of the internal friction of fluids on the motion of pendulums. Trans. Cambridge Philos. Soc, 9, 8-106.

Van de Ven, T. G. M. 1989. Colloidal hydrodynamics. London, Academic Press. 